\title{
Comparative transcriptome analysis of Glyphodes pyloalis Walker (Lepidoptera: Pyralidae) reveals novel insights into heat stress tolerance in insects
}

\author{
Yuncai Liu, Hang Su, Rongqiao Li, Xiaotong Li, Yusong Xu*, Xiangping Dai, Yanyan Zhou and Huabing Wang ${ }^{*}$
}

\begin{abstract}
Background: Heat tolerance is a key parameter that affects insect distribution and abundance. Glyphodes pyloalis Walker (Lepidoptera: Pyralidae) is a devastating pest of mulberry in the main mulberry-growing regions and can cause tremendous losses to sericulture by directly feeding on mulberry leaves and transmitting viruses to Bombyx mori. Moreover, G. pyloalis shows a prominent capacity for adaptation to daily and seasonal temperature fluctuations and can survive several hours under high temperature. To date, the molecular mechanism underlying the outstanding adaptability of this pest to high temperature remains unclear.

Results: In this study, we performed comparative transcriptome analyses on G. pyloalis exposed to 25 and $40{ }^{\circ} \mathrm{C}$ for $4 \mathrm{~h}$. We obtained 34,034 unigenes and identified 1275 and 1222 genes significantly upregulated or downregulated, respectively, by heat stress. Data from the transcriptome analyses indicated that some processes involved in heat tolerance are conserved, such as high expression of heat shock protein (HSP) genes and partial repression of metabolism progress. In addition, vitamin digestion and absorption pathways and detoxification pathways identified here provided new insights for the investigation of the molecular mechanisms of heat stress tolerance. Furthermore, transcriptome analysis indicated that immune and phosphatidylinositol signaling system have a close relationship with heat tolerance. In addition, the expression patterns of ten randomly selected genes, such as HSP and cytochrome P450, were consistent with the transcriptome results obtained through quantitative real-time PCR.
\end{abstract}

Conclusions: Comparisons among transcriptome results revealed the upregulation of HSPs and genes involved in redox homeostasis, detoxication, and immune progress. However, many metabolism progresses, such as glycolysis/ gluconeogenesis and fatty acid biosynthesis, were partially repressed. The results reflected that the heat tolerance of $G$. pyloalis is a fairly complicated process and related to a broad range of physiological regulations. Our study can improve understanding on the mechanisms of insect thermal tolerance.

Keywords: Glyphodes pyloalis, Heat stress, RNA-Seq, Heat shock protein, Redox homeostasis, Metabolism, Immune

\section{Background}

High temperature is an environmental factor that limits the distribution and abundance of insects. Insects are sensitive to temperature changes [1]. Over the past 30 years, global warming has led to significant changes in the number of insect species [2]. Several insect species, such as Drosophila melanogaster (D. melanogaster) and Bombyx mori (B. mori), are sensitive to heat stress,

\footnotetext{
* Correspondence: xuyusong@zju.edu.cn; wanghb@zju.edu.cn College of Animal Sciences, Zhejiang University, Hangzhou 310058, China
}

and the mass mortalities of these insects are often caused by high temperature [3, 4]. Conversely, many pests have evolved outstanding capability to adapt to high-temperature stimulations [5]. In recent years, many studies have focused on the effect of temperature on these insects because of their adaptability to a broad range of temperature [6].

Heat stress and acclimation of insect are currently considered a multistep process, involving a combination of behavioral, physiological, and cellular responses [7, 8] 
Recent studies showed that heat tolerance in certain organisms is related to protein folding, degradation, transport, and metabolism $[9,10]$. Moreover, current studies on flies revealed that several signal pathways, such as stress-responsive c-Jun N-terminal kinase signaling pathway, play an important role in adaptive metabolic response to heat stress [11]. Thus, many biologists have attempted to uncover the mechanisms underlying gene expression regulation under heat stress. Recently, researchers have characterized proteomic responses induced by heat stress in insects, such as Aphids, D. melanogaster, and B. mori. These studies displayed many proteindetermining thermotolerances, such as chromatin remodeling and translation, iron ion and cell redox homeostasis, and carbohydrate and energy metabolism [12-14]. Despite these findings, minimal information is available on the gene expression profile of pest resistant to heat stress.

Glyphodes pyloalis Walker (Lepidoptera: Pyralidae), a specialist pest on mulberry, is widely distributed throughout Asia. This pest can damage sericulture not only by feeding on mulberry but also by transmitting viruses to B. mori. G. pyloalis encounters a wide range of daily and seasonal temperature fluctuations. On a summer day, the average temperature in Hangzhou (120.2' E, 30.3' N), Zhejiang Province, China is about $33.8{ }^{\circ} \mathrm{C}$, and the highest temperature can reach almost $42{ }^{\circ} \mathrm{C}$. Temperature increases during the summer lead to adverse effects on temperate bivoltine silkworm rearing and cause economic losses. In contract, G. pyloalis adapt to relatively high temperatures $\left(35-40{ }^{\circ} \mathrm{C}\right)$ [15]. However, previous studies of G. pyloalis have focused only on single genes, such as $\alpha$ - and $\beta$-glucosidase [16]; the molecular mechanisms involved in the heat responses have not been explored through the transcriptome method, which has been widely used in profile gene expressions in insects. Uncovering the conundrum of the heat tolerance of G. pyloalis through RNASeq technique is reasonable, because the mechanism of $G$. pyloalis in managing heat stress remains unknown.

Midgut is the primary site of digestion and absorption in insects [17]. This site not only controls food storage and nutrient absorption but also maintains water, ion, and osmotic pressure balance [18]. Moreover, the midgut of an insect is involved in immunity and detoxication of harmful substances during digestion and absorption [19]. Thus, it is a pivotal organ for the interaction between an insect and external environment, and it represents a key target for the heat tolerance of an insect. However, few studies have focused on midgut transcriptome response to high temperature [20].

In the present study, G. pyloalis, an important lepidopteran pest, was assessed for the effects of heat acclimation. The results showed that approximately one-seventh of the transcriptome are differentially regulated upon heat acclimation. In addition, our results revealed that genes, which are related to heat tolerance, received pronounced effect after heat stimulation. This result showed that heat tolerance is a more complex process than we previously assumed. Our study can improve understanding on the molecular mechanism of heat tolerance in insects and provide novel targets for pest prevention and control.

\section{Results}

\section{Heat resistance of G. pyloalis Walker}

G. pyloalis and B. mori are specialist insects on mulberry. Morphological changes in the midguts of G. pyloalis and $B$. mori were monitored after heat stress. Histological staining was performed to determine whether G. pyloalis can elicit heat resistance in its histomorphology and structure level under high temperatures (Fig. 1). After exposure to $40{ }^{\circ} \mathrm{C}$ for $4 \mathrm{~h}$, the midgut of $\mathrm{B}$. mori had a weaker condition than that exposed at $25{ }^{\circ} \mathrm{C}$ (Fig. 1a and b). Heat stress resulted in significant changes, and a large number of bubble-like structures were observed adjacent to the midgut contents of B. mori (Fig. 1b). However, structures observed in the midgut of $G$. pyloalis were fewer than those in B. mori after heat stress (Fig. 1b and d).

mRNA sequencing, assembly, and functional annotation We performed RNA-Seq to quantify the expression of genes in G. pyloalis to elucidate the molecular basis of heat stress response. RNA was extracted from the midgut of G. pyloalis and the transcriptome was sequenced using Illumina short reads. Approximately $13.37 \mathrm{~Gb}$ data and 34,034 unigenes were obtained (Additional file 1). The total length, average length, N50, and GC content of unigenes were 36,437,658 bp; 1070 bp; 2000 bp; and 43.09\%, respectively (Table 1 ). We also detected the distinction of unigene length distributions between the two treatments and found no significant difference (Additional file 2). However, there were 22,729 unigenes expressed in the normal condition $\left(25^{\circ} \mathrm{C}\right)$ while 23,051 unigenes expressed after heat stress. We then annotated our unigenes with seven functional databases and found that 19,653 (57.75\%), 13,170 (38.70\%), 15,353 (45.11\%), 8354 (24.55\%), 15,546 (45.68\%), 3878 (11.39\%), and 14,410 (42.34\%) unigenes can be mapped to NR, NT, UniProtKB/Swiss-Prot (Swiss-Prot), Cluster of Orthologous Groups of proteins (COG), Kyoto Encyclopedia of Genes and Genomes (KEGG), Gene Ontology (GO), and Interpro databases, respectively. After performing functional annotation, we detected 19,609 CDS. The remaining unaligned unigenes were analyzed using ESTScan to predict the coding regions. Another 1821 CDS were obtained after the analysis. The G. pyloalis sequences showed $42.9 \%$ matches with B. mori, followed by Danaus plexippus (27.63\%), Papilio xuthus (2.2\%), and Riptortus pedestris (2.13\%) (Additional file 3). Furthermore, 2497 unigenes were 

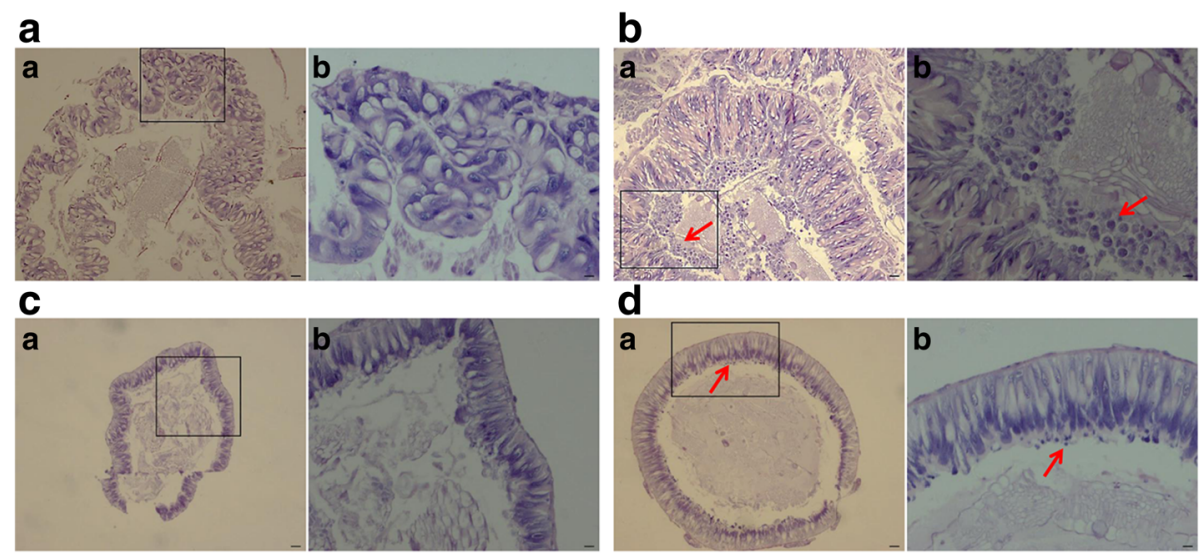

Fig. 1 Morphological changes of midgut with different treatments. Morphological changes of B. mori (A-B) and G. pyloalis (C-D) were observed. Each (a) is a picture with low magnification, and the blank-lined area is shown in (b) with high magnification. Minimal bubble-like structure was observed in G. pyloalis after $4 \mathrm{~h}$ of heat treatment. However, many bubble-like structures (indicated by arrowheads) were produced in B. mori. Bars: A-a, B-a, C-a, and D-a: $10 \mu \mathrm{m} ; \mathrm{A}-\mathrm{b}, \mathrm{B}-\mathrm{b}, \mathrm{C}-\mathrm{b}$, and D-a: $3.3 \mu \mathrm{m}$

differentially regulated after heat shock treatment with a criterion of $\mid$ fold change $\mid \geq 4.0$ and FDR $\leq 0.001$ in DEGs definition. Of these DEGs, 1275 genes were upregulated, and 1222 genes were downregulated (Fig. 2). Ten significantly most upregulated genes that response to heat stress are as follows: ribonuclease, thiolase 4, peroxisomal multifunctional enzyme type 2-like, keratin-associated protein 10-7-like, aldehyde dehydrogenase isoform 1, PGI4-45_10 phosphoglucose isomerase, Probable cytochrome P450 304a1, Purine nucleoside phosphorylase associated with the functions redox homeostasis, detoxication and many metabolisms progresses (Additional file 4). This analysis showed heat stress had significant effect on the gene expression.

\section{GO and KEGG analyses of DEGs}

We focused on the 1275 induced and 1222 repressed genes to further understand the overall biology of transcriptional response in G. pyloalis to heat stress. For GO analysis, we annotated DEGs into three GO categories, namely, cell component, molecular function, and biological process (Fig. 3). In the cell component category, the terms "glutamyl-tRNA amidotransferase complex" and "membrane" were the enriched components. "Steroid hormone receptor activity" and "lipid transporter activity"

Table 1 Distribution of differentially expressed unigenes and Quality metrics of Unigenes

\begin{tabular}{llllllll}
\hline Sample & $\begin{array}{l}\text { Total } \\
\text { Number }\end{array}$ & $\begin{array}{l}\text { Total } \\
\text { Length }\end{array}$ & $\begin{array}{l}\text { Mean } \\
\text { Length }\end{array}$ & N50 & N70 & N90 & GC (\%) \\
\hline Control & 27,703 & $27,143,070$ & 979 & 1789 & 987 & 365 & 43.42 \\
Heat-shock & 29,106 & $27,433,438$ & 942 & 1693 & 929 & 353 & 43.34 \\
All-Unigene & 34,034 & $36,437,658$ & 1070 & 2000 & 1146 & 397 & 43.09 \\
\hline
\end{tabular}

N50: $50 \%$ of the Total Length is contained in Unigenes greater than or equal to this value were the top two molecular function terms. The most enriched components of the biological process category were "steroid hormone mediated signaling pathway", "response to a steroid hormone" and "cellular response to steroid hormone stimulus".

KEGG is a database for biological systems that integrates genomic, chemical, and systemic functional information [21]. In this study, 1666 DEGs were mapped to 291 pathways. Among these DEGs, "metabolic pathways" was the most dominant pathway in the midgut. In addition, many pathways, such as "sphingolipid metabolism", "phenylalanine metabolism", "RIG-I-like receptor signaling pathway" and "Toll-like receptor signaling pathway" were also enriched (Additional file 5). These results indicated an integration between metabolic and immune responses during heat tolerance, and this integration ensures energy balance and permits growth and defense in G. pyloalis.

\section{Expression of pathways and genes for heat tolerance Heat shock proteins (HSPs)}

The transcriptional response of insects to heat shock includes a large number of HSPs, implying a universal mechanism of heat tolerance in insects [22]. We focused on genes that encoded HSPs to reveal the generality and particularity of heat tolerance between G. pyloalis and other insects. In this study, the data revealed upregulation or downregulation of HSPs under heat stress conditions. As shown in Fig. 4, 12 HSPs, including HSP70, HSP40, and small HSPs (sHSPs), which were distributed in different families, were identified (|fold change $\mid \geq 4.0$ and FDR $\leq$ 0.001). Among these HSPs, five genes belonged to the HSP70 family, whereas two and five genes belonged to the HSP40 and sHSP, respectively. In terms of gene expression levels, nine HSPs displayed greater than fourfold increased 


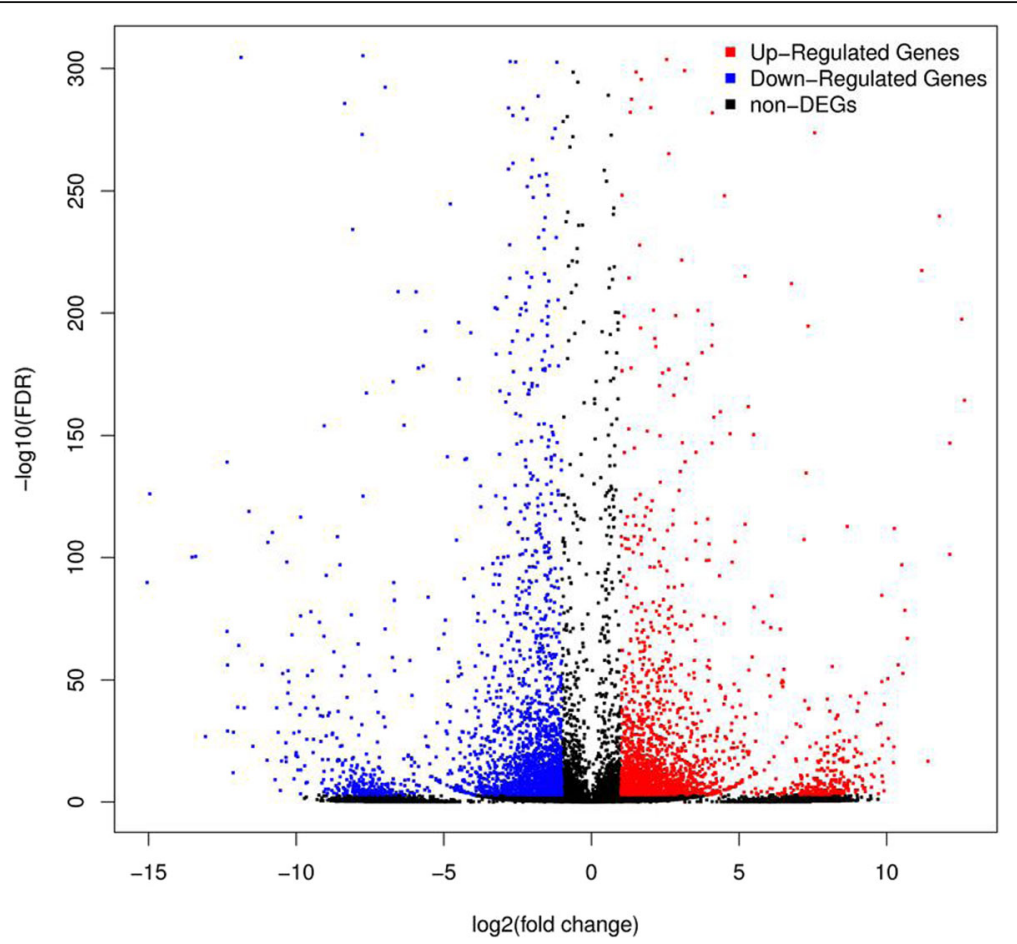

Fig. 2 DEGs in the midgut after different temperature treatments. The variability pattern was displayed by volcano plot. Y axis represents - log 10 significance. $X$ axis represents log2-fold change. Red points represent upregulated genes. Blue points represent downregulated genes based on the discriminative significance values ( $\mid$ fold change $\mid \geq 4.0$ and FDR $\leq 0.001$ ) adopted in this study

expression, indicating a vital role in protein processing and heat tolerance in G. pyloalis.

\section{Antioxidant and detoxication}

Apart from genes encoding HSPs, several genes, which are involved in antioxidant and detoxication and regulated by heat stress, were identified. These genes included genes encoding two superoxide dismutase (Unigene21826_All and CL573.Contig1_All), a peroxidase (CL1988.Contig2_All), and a thioredoxin (CL1904.Contig1_All). In addition, these genes are directly linked to the generation of reactive oxygen species (ROS) under heat stress [23, 24] (Table 2). Detoxication-related genes were also identified in our study, including genes encoding glutathione S-transferase (CL2634.Contig1_All, Unigene19946_All, Unigene21727 _All, and CL3231.Contig2_All) and cytochrome P450s (CYPs). 14 of the 23 cytochrome P450 (CYPs) unique sequences were induced, and most of these sequences were grouped into CYP3 clade (five of seven sequences), CYP6 clade (four of eight unique sequences), and CYP9 (three sequences; Fig. 5). Meanwhile, the polypeptides of the two unique sequences, namely, CL716.Contig3_All and Unigene19157_All, were determined as unigenes for aldehyde dehydrogenase (ALDH). They have been considered effective detoxifying enzymes and are involved in many fundamental biochemical pathways, including reduced cytotoxic aldehydes triggered by lipid peroxidation
[25]. Therefore, antioxidant and detoxication have a close relationship with heat tolerance and even share a similar handling mechanism.

\section{Metabolism and protein turnover}

Through KEGG pathway enrichment analysis, we found that many pathways related to metabolic reactions were enriched, including carbohydrate (14 pathways), lipid (15 pathways), and amino acid metabolisms (14 pathways). The genes associated with carbohydrate metabolism were partially repressed, such as glycolysis/gluconeogenesis (16 DEGs, 0.96\%), fructose and mannose metabolism (13 DEGs, $0.78 \%$ ), starch and sucrose metabolism (18 DEGs, $1.08 \%$ ), galactose metabolism (15 DEGs, 0.9\%), and oxidative phosphorylation (17 DEGs, 1.02\%). A repression in the processes involved in lipid metabolism, including sphingolipid metabolism (23 DEGs, 1.38\%), steroid biosynthesis (13 DEGs, 0.78\%), fatty acid degradation (21 DEGs, 1.26\%), and glycerolipid metabolism (25 DEGs, $1.5 \%)$, was also observed. The downregulated genes in fructose and mannose metabolism, glycolysis/gluconeogenesis, fatty acid biosynthesis, and fatty acid elongation pathways are illustrated in Fig. 6. Significant changes in metabolismrelated genes involved in heat response were consistent with a previous report describing global gene expressions in livestock [26, 27]. In addition, unigenes, which related to protein turnover processes, were induced in response to 


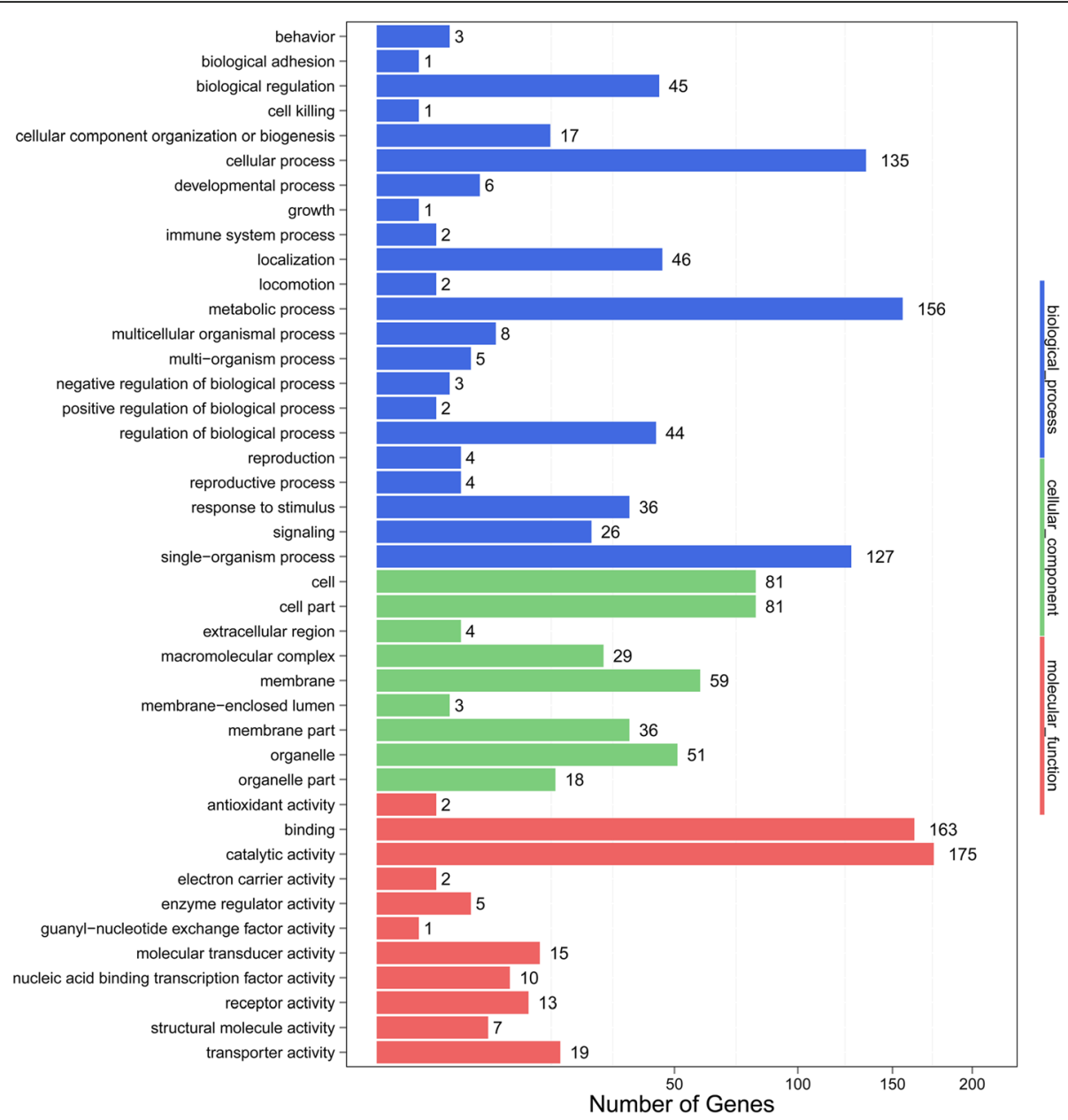

Fig. $3 \mathrm{GO}$ enrichment analysis revealed the biological processes most associated with detected DEGs. Based on the GO results, cellular progress, metabolic progress, binding, and catalytic activity were the most enriched $\mathrm{GO}$ terms under heat stress

heat stress. Among these unigenes, one proteasome-related unigene (CL2694.Contig1_All) was significantly upregulated. In addition, 34 of 57 ubiquitin system-related unigenes were specifically induced in response to heat stress, suggesting that the stress-related autophagy mechanisms were induced after heat stress. 8 of 10 ribosomal proteins, which were involved in protein translation and regulation of protein formation, were upregulated. These results suggested that protein turnover response was induced for protection against the detrimental effects of protein misfolding.

\section{Immune response}

Insects lack adaptive immunity and rely on innate immune reactions for their defense [28]. In the present study, a large number of immune-related genes were enriched, and most of the gene sets demonstrated an upregulated expression after treatment at $40{ }^{\circ} \mathrm{C}$. These genes were mainly involved in five pathways, namely, RIG-I-like receptor signaling pathway (8 DEGs, 0.48\%), Fc gamma R-mediated phagocytosis (44 DEGs, 2.64\%), chemokine signaling pathway (38 DEGs, 2.28\%), Toll-like receptor signaling pathway (12 DEGs, $0.72 \%$ ), and antigen processing and presentation (6 DEGs, 0.36\%) (Fig. 7a). Nine unigenes involved in the Toll-like receptor signaling pathway were upregulated and thus may play a central role in relaying intracellular immune signals (Fig. 7b). Meanwhile, some unigenes participating in immune defense mechanisms were also detected in our data, although they do not belong to the pathways mentioned previously. Three unigenes (CL1248.Contig8_All, Unigene8334_All, and Unigene6267_All) were found to encode lectin, which is directly associated with the component of the immune system. Five genes encoding lysozyme were also determined, and three of them (CL623.Contig1_All, Unigene6122_All, and Unigene5037 _All) had high expression levels and two (Unigene12321 _All and CL623.Contig2_All) had low expression levels. Furthermore, some antiviral-related genes, such as genes encoding hdd1 (Unigene19527_All), trypsin-like serine proteinase (CL1159.Contig4_All), and scavenger receptor 




Fig. 4 Comparative distribution of HSP coding genes between two samples. High temperature resulted in gene expression related to HSP70, HSP40, and small HSP. The color scale is displayed at the upper left, which encompasses from the lowest (green) to the highest (red) RPKM value

class B (Unigene7913_All), were induced. The abundant expression of immune response-related genes suggested that the immune responses were activated after heat shock.

\section{Stress signal transduction}

Stress signal transduction is the most important aspect for high temperature response. Many pathways involved in stress signal transduction were enriched in our analysis, such as Phosphatidylinositol (PI) signaling system (25 DEGs, 1.5\%), Notch signaling pathway (16 DEGs, 0.96\%), TNF signaling pathway (21 DEGs, 1.26\%), Phospholipase D signaling pathway (34 DEGs, 2.04\%),
Jak-STAT signaling pathway (15 DEGs, 0.9\%) and AMPK signaling pathway pathways (26 DEGs, 1.56\%) (Table 3). The threonine protein kinase (CL962.Contig1_All) was also upregulated, and its activation was responsible for cellular stresses, such as heat shock [29]. These findings indicate the importance of signal transduction in the thermal tolerance of G. pyloalis.

\section{Validation of data through quantitative real-time PCR (qRT-PCR)}

Thousands of genes showed significantly different expression levels. In this study, we randomly selected ten

Table 2 Changes in the transcriptional expression of redox homeostasis and detoxication-related genes in response to thermal stress $\left(40^{\circ} \mathrm{C}\right)$ for $4 \mathrm{~h}$

\begin{tabular}{|c|c|c|c|c|c|}
\hline Gene ID & Control & Heat shock & Regulated & $P$ value & $\overline{\text { Gene description }}$ \\
\hline CL1904.Contig1_All & 1.66 & 83.63 & up & 0 & Thioredoxin \\
\hline Unigene21826_All & 0.29 & 4.95 & up & 7.98E-09 & [Cu-Zn] SOD \\
\hline CL573.Contig1_All & 13.58 & 57.84 & up & 3.75E-84 & [Cu-Zn] SOD \\
\hline CL1988.Contig2_All & 2.37 & 16.18 & up & $6.73 \mathrm{E}-122$ & POD \\
\hline CL2634.Contig1_All & 0.01 & 3.84 & up & 1.45E-08 & GSTT1 \\
\hline Unigene19946_All & 0.01 & 2.78 & up & 4.64E-06 & GSTe4 \\
\hline Unigene21727_All & 0.17 & 3.37 & up & $1.24 \mathrm{E}-05$ & GSTu1 \\
\hline CL3231.Contig2_All & 1.05 & 4.24 & up & $1.16 \mathrm{E}-13$ & GSTT1 \\
\hline CL716.Contig3_All & 0.01 & 35.38 & up & $2.09 \mathrm{E}-242$ & $\mathrm{ALDH}$ \\
\hline Unigene19157_All & 1.04 & 4.91 & up & $2.02 \mathrm{E}-05$ & $\mathrm{ALDH}$ \\
\hline
\end{tabular}

Significant FDR $\leq 0.001$ for mRNA expression is represented. SOD: Superoxide dismutase; ALDH: Aldehyde dehydrogenase 

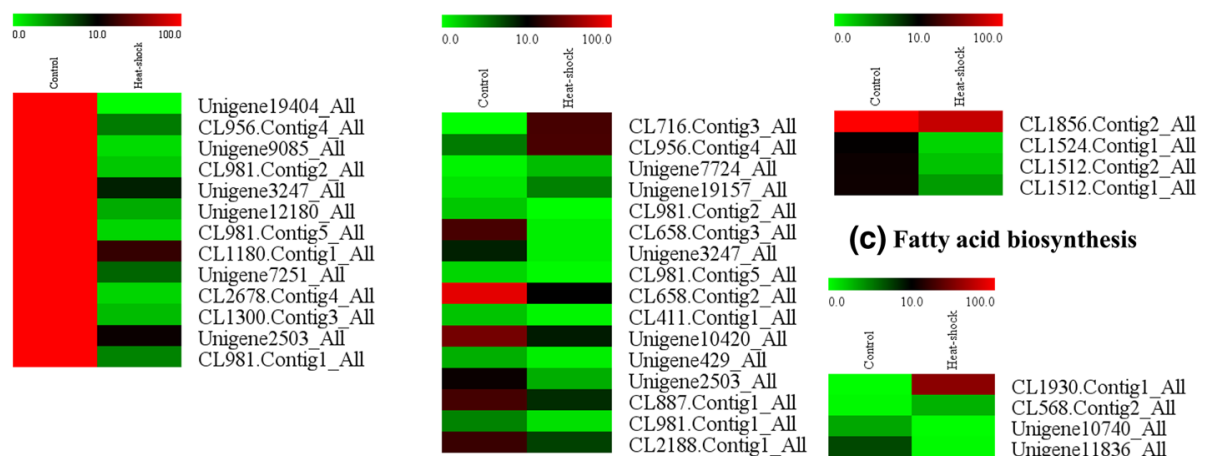

(c) Fatty acid biosynthesis

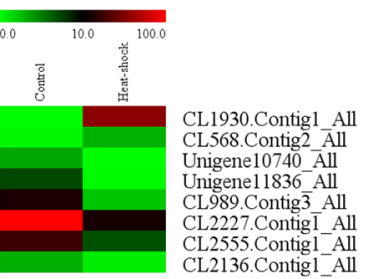

(a) Fructose and mannose metabolism (b) Glycolysis/guconeogenesis

(d) Fatty acid enlongation

Fig. 5 Heatmap of the expression level of different pathways. Expression level of some genes of fructose and mannose metabolism, glycolysis/ gluconeogenesis, fatty acid biosynthesis, and fatty acid elongation was shown in $\mathbf{a}, \mathbf{b}, \mathbf{c}$, and $\mathbf{d}$, respectively. The color scale is shown at the upper left, which encompasses from the lowest (green) to the highest (red) RPKM value

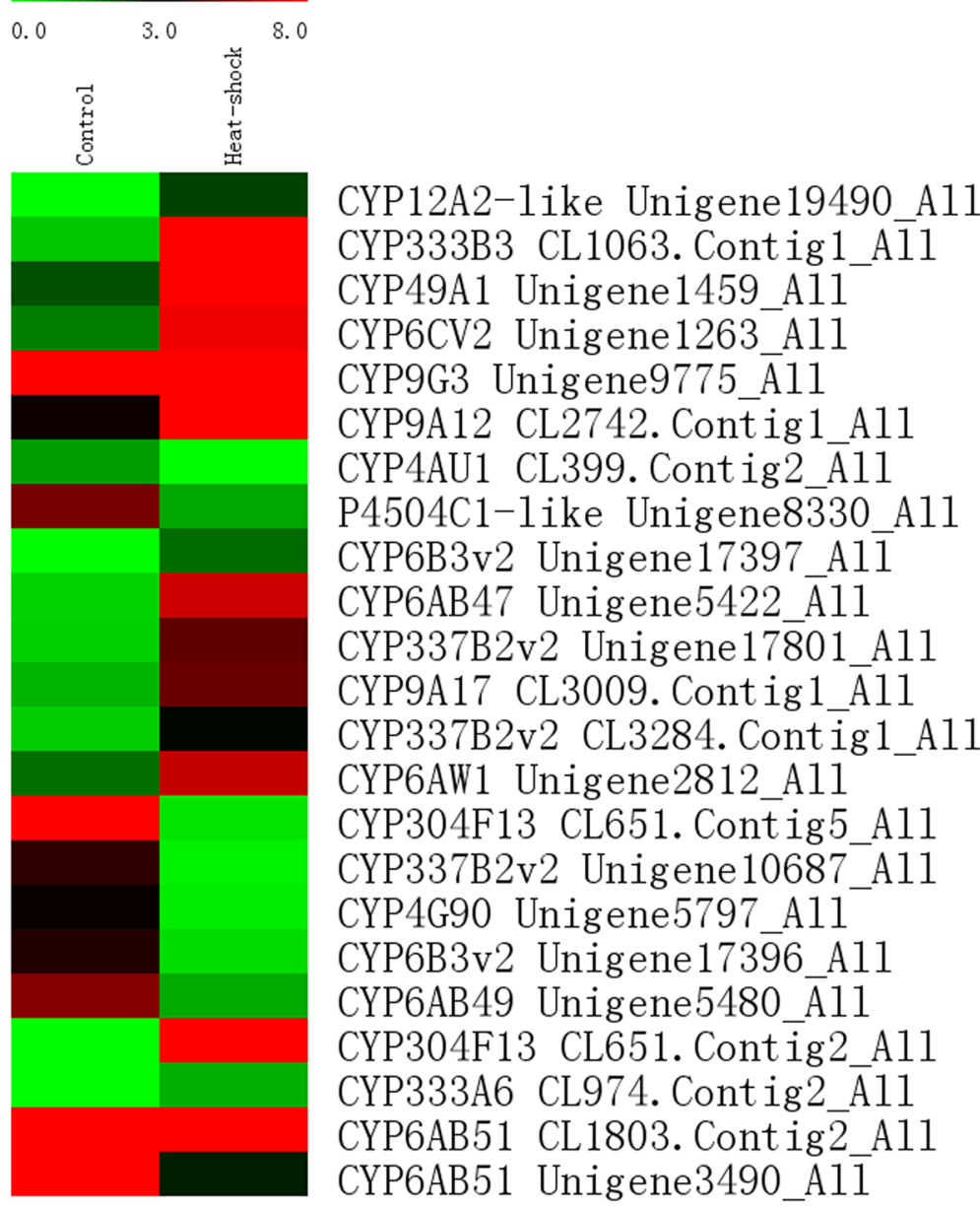

Fig. 6 Heatmap of the different expression of cytochrome P450 genes under different heat treatment temperatures. The color scale is shown at the upper left, which encompasses from the lowest (green) to the highest (red) RPKM value 


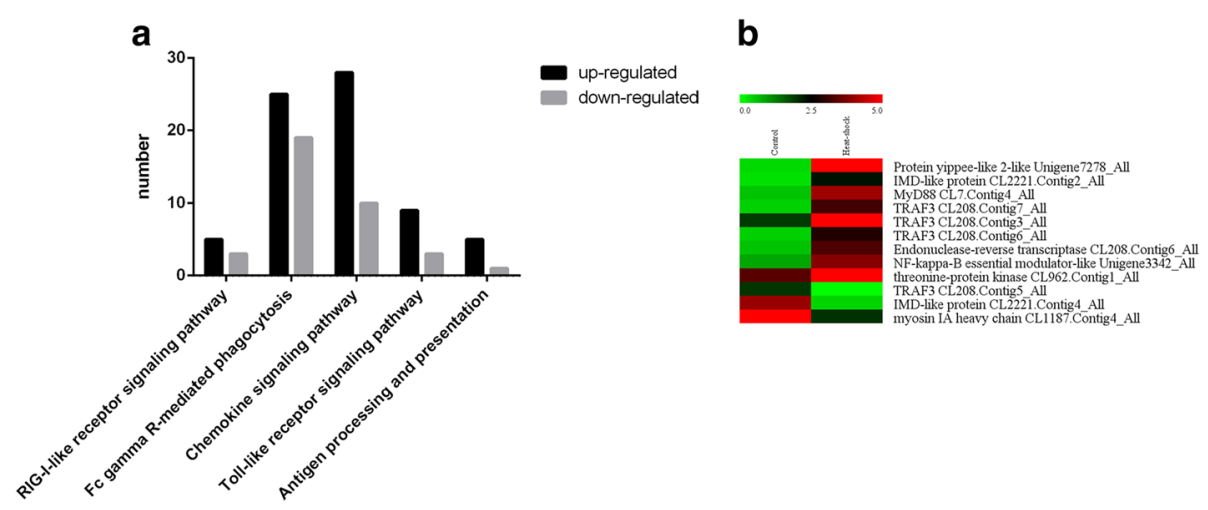

Fig. 7 General statistics on gene regulation under different temperatures and heatmap of the expression level of Toll-like receptor signaling pathways. a Numbers of upregulated and downregulated genes associated with various immune events. b Heatmap of genes related to Toll-like receptor signaling pathways. The color scale is shown at the upper left, which encompasses from the lowest (green) to the highest (red) RPKM value

genes to confirm their expression levels through qRT-PCR. Expression patterns were validated among the ten annotated transcripts (CL2742.Contig1_All, Unigene 4872_All, Unigene7931_All, CL658.Contig2_All, CL2 227.Contig1_All, Unigene5878_All, CL887.Contig1_All Unigene6953_All, Unigene6918_All and CL1060.Contig3 _All). Some genes related to heat tolerance showed upregulated expression levels at $40{ }^{\circ} \mathrm{C}$. These genes included genes encoding CYP9G3 (CL2742.Contig1_All), CYPB5 (Unigene6953_All), HSP19.7 (Unigene4872_All), and HSP16.1 (Unigene7931_All). Meanwhile, we found that Ribosomal protein L32 (Rpl32) and $\beta$-actin were stable. The Rpl32 was used as an internal control. The changing trend of the qRT-PCR results was consistent with the results obtained by DEG expression profiling (Fig. 8).

\section{Discussion}

Many insects develop various mechanisms, including morphological and physiological adaptations, to cope with high-temperature stress. In this study, the morphological features of G. pyloalis indicated heat tolerance superior to that of $B$. mori and thus can contribute to its effective survival under high temperature. The adaptation strategies of G. pyloalis to heat stress may also be related to the expression of some unique genes. We conducted high-resolution analysis on transcriptome dynamics to identify the genes associated with excellent heat tolerance of G. pyloalis. In total, we obtained 2497 DEGs (1275 upregulated and 1222 downregulated) under heat stress and analyzed a large suite of biomarkers related to antioxidant, detoxication, metabolism, protein turnover, immune, and stress signal transduction. The GO analysis results showed that most of the DEGs after heat treatment were significantly represented in "response to stimulus", "regulation of response to stimulus", "response to steroid hormone" and "cellular response to steroid hormone stimulus". This finding suggested that most of the DEGs at the early heat stress stages were mainly associated with stress response and similar experiment results were observed in the plant response to heat stress. Many genes associated with "membrane", which revealed that most of the cells required being repaired [30]. Moreover, many highly represented pathways, such as sphingolipid metabolism, phenylalanine metabolism, metabolic pathways, and PI signaling system, were enriched when exposed to heat stress, indicating the importance of these pathways in establishing thermal tolerance [31].

In insects, HSPs act as molecular chaperones and participate in various cellular processes, such as protein folding, proteolytic pathway, or stabilizing denatured proteins [32-34]. HSPs can be divided into several families, namely, HSP40, HSP60, HSP70, HSP90 and sHSPs according to their molecular weights [35]. Twelve HSPs distributed in the HSP70, HSP40, and sHSPs were

Table 3 Significantly enriched signal pathways after heat treatment

\begin{tabular}{|c|c|c|c|c|}
\hline Signal pathway & DEGs genes with pathway annotation & All genes with pathway annotation & $P$ value & Pathway ID \\
\hline Phosphatidylinositol signaling system & $25(1.5 \%)$ & $162(1.04 \%)$ & 0.03917114 & ko04070 \\
\hline Notch signaling pathway & $16(0.96 \%)$ & $94(0.6 \%)$ & 0.04112893 & ko04330 \\
\hline TNF signaling pathway & $21(1.26 \%)$ & $134(0.86 \%)$ & 0.04798497 & ko04668 \\
\hline Phospholipase D signaling pathway & $34(2.04 \%)$ & $239(1.54 \%)$ & 0.05239752 & ko04072 \\
\hline Jak-STAT signaling pathway & $15(0.9 \%)$ & $91(0.59 \%)$ & 0.05950994 & ko04630 \\
\hline AMPK signaling pathway & $26(1.56 \%)$ & $182(1.17 \%)$ & 0.07817966 & ko04152 \\
\hline
\end{tabular}




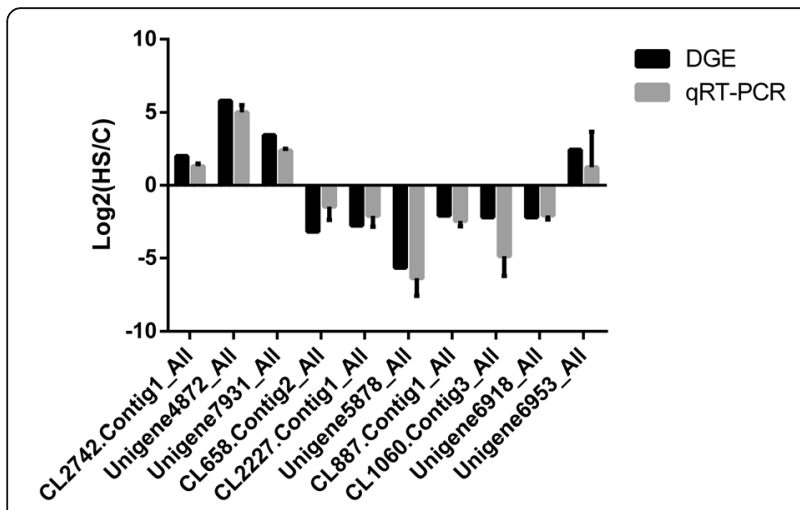

Fig. 8 qRT-PCR analysis of the expression levels of ten unigenes. $X$ axis represents different unigenes. CL2742.Contig1_All, cytochrome P450 9G3; Unigene4872_All, HSP 19.7; Unigene7931_All, HSP 16.1;

CL658.Contig2_All, aldehyde dehydrogenase family of seven members

A1; CL2227.Contig1_All, acetyltransferase 1; Unigene5878_All,

hemolymph juvenile hormone-binding protein; CL887.Contig1_All,

aldose 1-epimerase-like; CL1060.Contig3_All, 17-beta-hydroxysteroid

dehydrogenase; Unigene6918_All, Hematopoietically-expressed

homeobox protein HHEX homolog; Unigene6953_All, cytochrome P450

B5. Y axis represents the relative expression levels of genes. Ribosomal

protein L32 (Rp/32) was used as an internal control

observed to respond to heat treatment during the transcriptome analysis of G. pyloalis. The preferential induction of HSPs upon heat stress has been widely reported in numerous studies. In D. melanogaster, the accumulation of a major HSP, namely, HSP70, affects inducible thermal tolerance in larvae and pupae [36]. In Ceratitis capitata, expression of $\mathrm{Hsp} 70$ and thermal tolerance were assayed at a range of temperatures in several stages of its development. In the current study, the induced expression of five HSP70s in G. pyloalis possibly facilitated the refolding of damaged proteins and prevented protein aggregation under heat stress. In addition, five small HSPs of G. pyloalis were upregulated by heat stress and likely cooperated with co-chaperones for the prevention of cellular protein aggregation [37]. sHSPs are abundant in nearly all organisms and act as a response machine of organisms to some extreme stresses [38]. These results suggested that the induction of HSP was an evolutionarily conserved mechanism, and the coordinate upregulation of the molecular chaperones, including HSP70 and sHSP, was an important factor in acquiring thermal tolerance and can render the midgut cells of G. pyloalis resistant to heat stress.

Exposure to heat stress can result in ROS production, which can directly lead to a variety of toxic effects, including protein dysfunction, lipid peroxidation, and oxidative stress. In relation to signal transduction of ROS, we detected upregulation of several genes involved in vitamin digestion and absorption pathways. Many studies have demonstrated the importance of vitamins in oxidative stress responses. Sies [39] mentioned that vitamins $\mathrm{C}$ and $\mathrm{E}$ can react with free radicals and singlet molecular oxygen, which is the basis for their functions as antioxidants. Notablely, the induction of vitamin digestion and absorption pathway may suggest a specific function in the adaption of insect to high temperature. To the best of our knowledge, this result is some of the first evidence indicating the existence of vitamin digestion and absorption pathway after heat stress in insects. In Table 2, several antioxidant-related genes, including superoxide dismutase (SOD), peroxidase (POD), and thioredoxin were strongly upregulated after $40{ }^{\circ} \mathrm{C}$ treatment. Recent proteomic analysis showed that SOD overexpression upregulated ROS scavenging in rice grains under heat stress. The SOD knockout rice was susceptible to heat stress, which suggested that SOD played an important role in adaptation to heat stress [40]. POD, as a regulator of ROS scavenger, is a hematin-containing oxidase that can catalyze the oxidation of reduced compounds [41]. Meanwhile, thioredoxin can be potentially responsive to ROS and can catalyze electron transport to ribonucleotide reductase and other reductive enzymes and transcription factors [42]. The coordinate upregulation of the three antioxidant-related genes after $40{ }^{\circ} \mathrm{C}$ treatment suggested that they were regulators of ROS scavenger in G. pyloalis and were potentially generate ROS in response to heat stress. Similar antioxidantrelated genes were also observed in Bactrocera dorsalis and Bemisia tabaci exposed to high temperatures [43, 44]. Our data indicated that a link between oxidative stress and vitamin digestion and absorption was present in heat tolerance of G. pyloalis.

In addition to ROS generation, heat stress can promote toxic substance accumulation. In this study, we found that many detoxication-related genes, such as genes coding CYP, glutathione-S-transferase (GST), and ALDH, showed upregulated mRNA expression patterns after $4 \mathrm{~h}$ of heat stress. In most species, CYP effectively catalyzes lipid peroxidation. Meanwhile, the induction of a state of oxidative stress plays a central role in CYP-dependent cytotoxicity [45]. Apart from eliminating drugs, cytochrome P450s catalyze a broad range of oxidative processes involved in the metabolism of fatty acids and biosynthesis of sterols. Therefore, the induction of 14 CYPs in G. pyloalis may be an essential step in heat tolerance. In addition, we found 3 of 5 CYPs in B. mori were significantly induced at $40{ }^{\circ} \mathrm{C}$, suggesting several CYPs played a conserved role in response to heat stress (Additional file 6). However, further experiments are necessary to determine the exact function of these proteins. Meanwhile, GSTs comprise a family of isozymes and can convert toxic substances into reduced toxic metabolites through chemical reactions involving the conjugation of glutathione [46]. They protect macromolecules from reactive electrophiles, such as environmental 
carcinogens, ROS products, and chemotherapeutic agents [46]. Our observation suggested that the upregulation of genes encoding GST was related to increased intracellular oxidative stress and cellular toxic substance metabolism [47]. Detoxification pathways identified here provided new insights for the investigation of the molecular mechanisms of heat stress tolerance. ALDHs can eliminate toxic aldehydes by catalyzing their oxidation to nonreactive acids, the enzyme activities of which is mainly implicated in the metabolism of endogenous lipid peroxidation products [48]. Two mRNAs for ALDH were significantly upregulated after heat shock, suggesting they contribute to the improved heat tolerance through metabolic pathway.

Insects have developed a range of strategies, such as metabolism adaptation, to manage heat stress. Our results indicated that the metabolism of G. pyloalis in high temperature was weak. A similar reduction was observed in livestock [27]. The suppression of metabolism progress may be a conservative progress and can reflect cellular homeostasis or an energy-saving mechanism to manage heat stress, because ATP generation is a highlyregulated process involving three metabolism pathways, namely, glycolysis, tricarboxylic acid cycle, and oxidative phosphorylation. In addition, the ROS generated by heat stress can chemically modify proteins and alter their biological functions. Therefore, the removal of damaged proteins is vital for the maintenance of cellular homeostasis. Numerous studies have revealed that the ubiquitinproteasome system plays a vital role in recognizing and degrading damaged proteins $[49,50]$. In the present study, 34 ubiquitin-related unigenes were induced by heat stress. These results revealed that the upregulation of protein degradation progress was involved in stress response and adaptation.

For insects, the innate immune system is the major effector response system [51]. In our results, the activities of the RIG-I-like and Toll-like receptor signaling pathways were modulated by heat stress. The role of transcriptional activation of the Toll-like receptor in heat shock response has been reported [52-54]. Several reports have described some endogenous ligands, such as HSP60 and HSP90, that activate TLR4 to regulate innate immune responses and are also induced by high temperature [55]. Frequent confrontation of the immune system with HSPs can potentiate immunity. The upregulation of lectin, lysozyme, $H d d 1$, trypsin-like serine proteinase, and scavenger receptor class B under high temperatures further suggested that heat stress facilitated the components of immune defense.

The sensing and transduction of intracellular stress signal are critical for the adaptation and survival of insects under heat stress. In plants, the involvement of calcium and calcium-activated calmodulin in heat shock signal transduction was already investigated [56].
However, the mechanisms that enable insects to sense heat signal and trigger intracellular responses remain unclear. The PI signaling system is vital during the progress of plant development and growth and associated with cellular responses to environmental stress [57]. Meanwhile, Notch signaling pathway participates in physiological and stress erythropoiesis [58]. In this study, the preferential induction of PI signaling system and Notch signaling pathway under heat stress was uncovered and the results suggested that gene expression in the PI signaling system was related to the heat tolerance mechanism of G. pyloalis. These results suggested that the PI signaling system and Notch signaling pathway rapidly and effectively regulated downstream signaling and gene expression in G. pyloalis in response to hightemperature stress.

\section{Conclusions}

In the present study, we presented the first comprehensive evaluation of the midgut transcriptome of G. pyloalis by using Illumina sequencing technology and conducted a comparative expression analysis after heat treatment. A total of 34,034 unigenes, which might provide a major genomic resource for investigating the midgut of $G$. pyloalis, were obtained. Most of these genes had an annotation with matches in the seven functional databases. Furthermore, 2497 DEGs were identified in the midgut after heat stress. The results of the GO and KEGG pathway enrichment analyses indicated that the DEGs related to metabolism, immunity, and signal transduction were enriched after heat stress. In addition, the transcriptome results revealed a number of genes that are potentially relevant to HSPs, antioxidant, and detoxication in G. pyloalis. These genes could be major targets for thermal tolerance. Many of which can be understood with deep functional studies. Nonetheless, our study provides insight into the heat tolerance of insects.

\section{Methods \\ Biological materials and tissue collection}

G. pyloalis larvae were collected from the mulberry fields of Zhejiang University, Hangzhou (120.2' E, 30.3' $\mathrm{N})$, Zhejiang Province, China. B. mori (N4) larvae were reared as described previously [59]. The method of high temperature treatments or thermal exposure was modified from methods of Xiao et al. (2016), Li et al. (2014) and Moallem et al. (2017) [20, 60, 61]. G. pyloalis and B. mori treated at $25{ }^{\circ} \mathrm{C}$ served as controls. For treatments, larvae on day 3 of the fifth instar were selected and separated into two groups with 13 larvae each. Larvae were reared in different chambers at $25^{\circ} \mathrm{C}$ and $40{ }^{\circ} \mathrm{C}$ for $4 \mathrm{~h}$, respectively. They were supplied hourly with fresh mulberry leaves. Three biological replications were performed with each treatment. Four males and four 
females were selected for each treatment. Clean midgut samples were collected from eight (four males and four females) G. pyloalis and B. mori, respectively. The sample were freezed at $-80{ }^{\circ} \mathrm{C}$ immediately.

\section{Histological staining}

After being exposed to different temperature treatments as described above, the samples of larval G. pyloalis and B. mori were collected for hematoxylin-eosin (HE) staining. The midgut of G. pyloalis was dissected and fixed in formalin-acetic acid-alcohol (FAA) liquid for $12 \mathrm{~h}$. The samples were dehydrated through a series of graded ethanol baths $(70 \%-100 \%)$ to displace water, and then infiltrated with paraffin wax, a finally cut into sections $(5 \mu \mathrm{m})$. The obtained tissue sections were stained with $2 \%$ Mayer's hematoxylin and 1\% eosin [62]. Slides were observed and image can be examined directly in the microscope (Nikon Nis-Elements).

\section{De novo assembly and gene annotation}

Total RNA was extracted using RNAiso plus reagent (TaKaRa) according to the manufacturer's protocol. The concentration and quality of total RNA was quantified using NanoDrop Spectrophotometer (Thermo Fisher Scientific). For each group, total RNA from three replicates was pooled together in equal quantities. Approximately $6 \mu \mathrm{g}$ of RNA representing each group were used for illumine sequence. After the highthroughput sequencing (Illumina HiSeq 4000) of midgut samples, we used Trinity to filter raw reads by discarding low-quality, adaptor-polluted, and high-content unknown base reads and to generate $150 \mathrm{bp}$ paired-end read lengths. The clean reads were assembled into primary transcripts, and overlapping transcripts were assembled into large contigs after removing redundant transcripts. The final unigenes were obtained through gene family clustering with Tgicl [63]. Gene function was annotated based on the following databases: NCBI nonredundant protein sequences (NR); InterPro member database; Clusters of Orthologous Groups of proteins (KOG/COG); Swiss-Prot (a manually annotated and reviewed protein sequence database); Kyoto Encyclopedia of Genes and Genomes (KEGG); and Gene Ontology (GO). The unigenes that were not aligned to any database mentioned above were predicted by ESTScan (http://sourceforge.net/projects/estscan).

\section{Gene expression quantification}

Bowtie2 software (http://bowtie-bio.sourceforge.net/Bowtie2/index.shtml) was used to map all the clean reads to the unigene library. Gene expression level was then calculated using the RSEM software (http://deweylab.biostat.wisc.edu/ RSEM). The fragments per kilobase of transcript per million (FPKM) mapped reads can be used to quantify the unigene expression level. False discovery rate (FDR) was used to determine the threshold $P$-value in multiple tests. Furthermore, |fold change $\mid \geq 4.0$ and FDR $\leq 0.001$ were used as the parameters for determining significant differences in gene expression. The DEGs were then used for GO and KEGG enrichment analyses. The $P$-value calculating formula in the hypergeometric test is:

$$
P=1-\sum_{i=0}^{\mathrm{m}-1} \frac{\left(\begin{array}{c}
M \\
\mathrm{i}
\end{array}\right)\left(\begin{array}{c}
N-M \\
n-i
\end{array}\right)}{\left(\begin{array}{c}
N \\
n
\end{array}\right)}
$$

In this equation, $\mathrm{N}$ and $\mathrm{n}$ indicate the number of genes with GO/KEGG annotations and the number of DEGs in $\mathrm{N}$, respectively. The variables $\mathrm{M}$ and $\mathrm{m}$ represent the numbers of genes and DEGs, respectively, in each GO/ KEGG term.

\section{qRT-PCR validation}

Ten annotated unigenes and five $B$. mori CYPs were randomly selected to quantify by qRT-PCR and evaluate data level. RNAiso plus reagent (TaKaRa) was used to extract total RNAs from three biological replications, and Primer Premier 5.0 was used to perform the primer design. qRT-PCR primers are displayed in Additional file 7 and Additional file 8 . The reverse transcription system was used to synthesize cDNA as described previously [64-66]. qRT-PCR was performed on a LightCycler ${ }^{\circ} 480$ real-time PCR system (Roche). The reaction system consisted of $2 \mu \mathrm{l}$ of cDNA, $10 \mu \mathrm{l}$ of SYBR Green qPCR master mix (TaKaRa), $0.5 \mu \mathrm{l}$ of each of the primers, and sterile water made up to $20 \mu \mathrm{l}$. The $\Delta \Delta \mathrm{Ct}$ method was used to analyze the relative differences in transcript levels [67]. Ribosomal protein L32 (Rpl32) was used for internal control, and the experiment was performed on three biological replicates.

\section{Additional files}

\section{Additional file 1: Distribution of base content and quality.} (DOCX $18 \mathrm{~kb}$ )

Additional file 2: Unigene length distribution in G. pyloalis midgut with different treatments. $X$ axis represents the length of unigenes. $Y$ axis represents the number of unigenes. A: "Control" unigene length distribution. B: "Heat shock" unigene length distribution. (TIFF 1807 kb)

Additional file 3: Distribution of annotated species. (TIFF $5077 \mathrm{~kb}$ ) Additional file 4: List of the significantly up- and down-regulated genes. (XLSX 570 kb)

Additional file 5: Pathway functional enrichment of DEGs. Based on the KEGG results, the metabolic pathway was the most enriched KEGG pathway under heat stress. (TIFF $1458 \mathrm{~kb}$ )

Additional file 6: qRT-PCR analysis of the expression levels of five randomly selected $B$. mori genes. (TIFF $61 \mathrm{~kb}$ )

Additional file 7: Primers used for B. mori CYPs. (DOCX $15 \mathrm{~kb}$ ) Additional file 8: Primers used for G. pyloalis. (DOCX 19 kb) 


\section{Abbreviations}

17 beta-HSD: 17-beta-hydroxysteroid dehydrogenase; A1E: aldose 1-epimerase-like; ALDH: Aldehyde dehydrogenase; AT: acetyltransferase 1; BLAST: Basic local alignment search tool; COG: Clusters of Orthologous Groups; CYP: cytochrome P450; DEGs: Differentially expressed genes; FAA: formalin-acetic acid-alcohol; FPKM: Fragments Per Kilobase of transcript per Million; GO: Gene ontology; GST: glutathione-S-transferase; HE: hematoxylin-eosin; Hhex: Hematopoietically-expressed homeobox protein; HSP: heat shock protein; JHBP: hemolymph juvenile hormone-binding protein; KEGG: Kyoto Encyclopedia of Genes and Genomes; NCBI: National Center for Biotechnology Information; NR: NCBI non-redundant protein database; Nt: Nucleotide sequence database; PI: Phosphatidylinositol; POD: peroxidase; qRT-PCR: Quantitative real-time PCR; RNA-Seq: RNA-sequencing; ROS: Reactive oxygen species; Rpl32: Ribosomal protein L32; SOD: superoxide dismutase; Swiss-Prot: UniProtKB/Swiss-Prot; TCA: tricarboxylic acid cycle

\section{Acknowledgements}

We thank Pengfei Zhan and Qiujie Qian for technical assistance.

\section{Funding}

This work was supported by the National Natural Science Foundation of China (Grant No. 31572321; 31,602,010). Sequencing library creation and high throughput sequencing was supported by a grant from the Science Foundation of Zhejiang Province of China (No. LY15C170001) and the public project grant (2016C32005) from the Science and Technology Department of Zhejiang Province.

\section{Availability of data and materials}

All data analyzed during this study are provided in this published article and additional files. The sequence data of this study have been deposited into Sequence Read Archive (http://www.ncbi.nlm.nih.gov/sra; accession number PRJNA385884)

\section{Authors' contributions}

YCL, HBW and YSX conceived this study. YCL, HS, YYZ and HBW collected data and data analyses. YCL and XTL contributed analysis tools. YCL, RQL, XPD and HBW carried out experiments. YCL and HBW wrote the manuscript with help from all the authors. All authors read and approved the final manuscript.

\section{Ethics approval and consent to participate} Not applicable.

\section{Consent for publication}

Not applicable.

\section{Competing interests}

I confirm that I have read BioMed Central's guidance on competing interests and have included a statement in the manuscript on any competing interests. The authors declare no competing financial interests.

\section{Publisher's Note}

Springer Nature remains neutral with regard to jurisdictional claims in published maps and institutional affiliations.

Received: 10 May 2017 Accepted: 28 November 2017 Published online: 19 December 2017

\section{References}

1. Hallman GJ, Denlinger DL: Temperature sensitivity in insects and application in integrated pest management. 1998

2. Zhang $\mathrm{G}$, Xingliang $\mathrm{XU}$, Zhou $\mathrm{C}$, Zhang $\mathrm{H}$, Hua O. Responses of vegetation changes to climatic variations in Hulun Buir grassland in past 30 years. Acta Geograph Sin. 2011;66(1):47-58.

3. Hercus MJ, Loeschcke V, Rattan SIS. Lifespan extension of Drosophila Melanogaster through hormesis by repeated mild heat stress. Biogerontology. 2003;4(3):149-56.

4. Manjunatha HB, Rajesh RK, Aparna HS. Silkworm thermal biology: A review of heat shock response, heat shock proteins and heat acclimation in the domesticated silkworm, Bombyx mori. J Insect Sci. 2010;10(204):204.
5. Pieterse W, Terblanche JS, Addison P. Do thermal tolerances and rapid thermal responses contribute to the invasion potential of Bactrocera Dorsalis (Diptera: Tephritidae)? J Insect Physiol. 2017;98:1-6.

6. Jesus TF, Grosso AR, Almeida-Val VMF, Coelho MM. Transcriptome profiling of two Iberian freshwater fish exposed to thermal stress. J Therm Biol. 2016; 55:54-61.

7. Garrad R, Booth DT, Furlong MJ. The effect of rearing temperature on development, body size, energetics and fecundity of the diamondback moth. B ENTOMOL RES. 2016;1(2):1-7.

8. Damos P, Savopoulou-Soultani M. Temperature-driven models for insect development and vital thermal requirements. Psyche. 2011;2012(1-2):1-13.

9. Kregel KC. Heat shock proteins: modifying factors in physiological stress responses and acquired thermotolerance. J Appl Physiol. 2002;92(5): 2177-86.

10. Zhang Y. Transcriptome responses to heat- and cold-stress in ladybirds (Cryptolaemus Montrouzieri Mulasnt) analyzed by deep-sequencing. Biol Res. 2015:48(1):66

11. Verheij M, Ruiter GA, Zerp SF, van Blitterswijk WJ, Fuks Z, Haimovitzfriedman A, Bartelink $H$. The role of the stress-activated protein kinase (SAPK/JNK) signaling pathway in radiation-induced apoptosis. Radiother Oncol. 1998; 47(3):225-32.

12. Nguyen TTA, Michaud D, Cloutier C. A proteomic analysis of the aphid Macrosiphum Euphorbiae under heat and radiation stress. Insect Biochem Mol Biol. 2009;39(1):20-30.

13. Colinet H, Overgaard J, Com E, Sørensen JG. Proteomic profiling of thermal acclimation in Drosophila Melanogaster. Insect Biochem Mol Biol. 2013; 43(4):352.

14. Li J, Ye L, Lan T, Yu M, Liang J, Zhong B. Comparative proteomic and phosphoproteomic analysis of the silkworm (Bombyx Mori) posterior silk gland under high temperature treatment. Mol Biol Rep. 2012;39(8):8447-56.

15. Ramegowda GK, Illahi I, Mittal V, Akhter I, Dhar A, Khan MA. Influence of weather on the incidence and severity of lesser mulberry Pyralid and mulberry Looper in Kashmir, India. J Entomol. 2012;9(6):422-8.

16. Ghadamyari M, Hosseininaveh V, Sharifi M: Partial biochemical characterization of alpha- and beta-glucosidases of lesser mulberry pyralid, Glyphodes pyloalis Walker (Lep.: Pyralidae). CR BIOL 2010, 333(3):197-204.

17. Swevers L, Huvenne H, Menschaert G, Kontogiannatos D, Kourti A, Pauchet Y, Ffrench Constant R, Smagghe G. Colorado potato beetle (Coleoptera) gut transcriptome analysis: expression of RNA interference-related genes. Insect Mol Biol. 2013;22(22):668-84.

18. Lei Y, Zhu X, Xie W, Wu Q, Wang S, Guo Z, Xu B, Li X, Zhou X, Zhang Y. Midgut transcriptome response to a cry toxin in the diamondback moth, Plutella xylostella (Lepidoptera: Plutellidae). Gene. 2014;533(1):180.

19. Valencia A, Wang H, Soto A, Aristizabal M, Arboleda JW, Eyun SI, Noriega DD, Siegfried B. Pyrosequencing the Midgut Transcriptome of the banana weevil Cosmopolites Sordidus (Germar) (Coleoptera: Curculionidae) reveals multiple protease-like transcripts. PLoS One. 2016:11(3):1-16.

20. Li QR, Xiao Y, Wu FQ, Ye MQ, Luo GQ, Xing DX, Li L, Yang Q. Analysis of midgut gene expression profiles from different silkworm varieties after exposure to high temperature. Gene. 2014;549(1):85.

21. Ogata H, Goto S, Sato K, Fujibuchi W, Bono H, Kanehisa M. KEGG: kyoto encyclopedia of genes and genomes. Nucleic Acids Res. 2000;27(1):29-34.

22. Lang RP, Bayne CJ, Camara MD, Cunningham C, Jenny MJ, Langdon CJ. Transcriptome profiling of selectively bred Pacific oyster Crassostrea Gigas families that differ in tolerance of heat shock. Mar Biotechnol. 2009;11(5): 650-68.

23. Imlay JA. Cellular defenses against superoxide and hydrogen peroxide. BIOCHEMISTRY-US. 2008;77(77):755-76.

24. Lu J, Holmgren A. The thioredoxin antioxidant system. Free Radic Biol Med. 2014;66(2):75-87.

25. Kotchoni SO, Jimenezlopez JC, Kayodé AP, Gachomo EW, Babamoussa L. The soybean aldehyde dehydrogenase (ALDH) protein superfamily. Gene. 2012;495(2):128-33.

26. Neven LG. Physiological responses of insects to heat. Postharvest Biol Technol. 2000;21(1):103-11.

27. Belhadj SI, Najar T, Ghram A, Abdrrabba M. Heat stress effects on livestock: molecular, cellular and metabolic aspects, a review. J Anim Physiol A Anim Nutr. 2015:100(3):401.

28. Cui Y, Hao Y, Li J, Bao W, Li G, Gao Y, Gu X. Chronic heat stress induces immune response, oxidative stress response, and apoptosis of finishing pig liver: a proteomic approach. Int J Mol Sci. 2016;17(5):393. 
29. Konishi H, Matsuzaki H, Tanaka M, Takemura Y, Kuroda S, Ono Y, Kikkawa U: Activation of protein kinase B (Akt/RAC-protein kinase) by cellular stress and its association with heat shock protein Hsp27. Febs Letters. 1997;410(2-3): 493-98.

30. Howard AC, Mcneil AK, Mcneil PL. Promotion of plasma membrane repair by vitamin E. Nat Commun. 2011;2(1):597.

31. Ivanina AV, Taylor C, Sokolova IM. Effects of elevated temperature and cadmium exposure on stress protein response in eastern oysters Crassostrea Virginica (Gmelin). Aquat Toxicol. 2009;91(3):245.

32. Frydman J. Folding of newly translated proteins in vivo: the role of molecular chaperones. Annu Rev Biochem. 2001;70(70):603-47.

33. Hartl FU, Hayerhartl M. Molecular chaperones in the Cytosol: from nascent chain to folded protein. Science. 2002;295(5561):1852-8.

34. Glickman MH, Ciechanover A. The ubiquitin-proteasome proteolytic pathway: destruction for the sake of construction. Physiol Rev. 2002;82(2): 373-428.

35. Nair S, Arora S, Lim JY, Lee LH, Lim LHK. The regulation of TNFa production after heat and endotoxin stimulation is dependent on Annexin-A1 and HSP70. Cell Stress Chaperones. 2015:20(4):583-93.

36. Feder ME, Cartaño NV, Milos L, Krebs RA, Lindquist SL. Effect of engineering Hsp70 copy number on Hsp70 expression and tolerance of ecologically relevant heat shock in larvae and pupae of Drosophila Melanogaster. J Exp Biol. 1996;199(Pt 8):1837.

37. Zhang J, Liu B, Li J, Zhang L, Wang Y, Zheng H, Lu M, Chen J. Hsf and Hsp gene families in Populus: genome-wide identification, organization and correlated expression during development and in stress responses. BMC Genomics. 2015;16(1):181.

38. Sarkar NK. Rice sHsp genes: genomic organization and expression profiling under stress and development. BMC Genomics. 2009;10(1):393.

39. Sies H, Stahl W. Vitamins E and C, beta-carotene, and other carotenoids as antioxidants. Am J Clin Nutr. 1995;62(6 Suppl):1315S.

40. Shiraya T, Mori T, Maruyama T, Sasaki M, Takamatsu T, Oikawa K, Itoh K, Kaneko K, Ichikawa H, Mitsui T. Golgi/plastid-type manganese superoxide dismutase involved in heat-stress tolerance during grain filling of rice. Plant Biotechnol J. 2015;13(9):1251-63.

41. Pirillo S, Ferreira ML, Rueda EH. Eriochrome blue black $R$ and Fluorescein degradation by hydrogen peroxide oxidation with horseradish peroxidase and hematin as biocatalysts. J Mol Catal B Enzym. 2010;66(1-2):63-71.

42. Ferguson AD, Labunskyy VM, Fomenko DE, Araç D, Chelliah Y, Amezcua CA, Rizo J, Gladyshev VN, Deisenhofer J. NMR structures of the selenoproteins Sep15 and SelM reveal redox activity of a new thioredoxin-like family. J Biol Chem. 2006;281(6):3536-43.

43. Mahadav A, Kontsedalov S, Czosnek H, Ghanim M. Thermotolerance and gene expression following heat stress in the whitefly Bemisia Tabaci B and Q biotypes. Insect Biochem Molec. 2009;39(10):668-76.

44. Wei D, Jia F, Tian C, Tian Y, Smagghe G, Dou W, Wang J. Comparative proteomic analysis of Bactrocera Dorsalis (Hendel) in response to thermal stress. J Insect Physiol. 2015;74:16-24.

45. Li X, Baudry J, Berenbaum MR, Schuler MA. Structural and functional divergence of insect CYP6B proteins: from specialist to generalist cytochrome P450. P Natl Acad Sci USA. 2004;101(9):2939-44.

46. de Aguiar ES, Giacomazzi J, Schmidt AV, Bock H, Saraiva-Pereira ML, SchulerFaccini L, Duarte FD, Dos Santos PA, Giugliani R, Caleffi M. GSTM1, GSTT1, and GSTP1 polymorphisms, breast cancer risk factors and mammographic density in women submitted to breast cancer screening. Rev Bras Epidemiol. 2012;15(2):246-55.

47. Shi H, Pei L, Gu S, Zhu S, Wang Y, Zhang Y, Li B. Glutathione S-transferase (GST) genes in the red flour beetle, Tribolium Castaneum, and comparative analysis with five additional insects. Genomics. 2012;100(5):327-35.

48. Pappa A, Estey T, Manzer R, Brown D, Vasiliou V. Human aldehyde dehydrogenase 3A1 (ALDH3A1): biochemical characterization and immunohistochemical localization in the cornea. Biochem J. 2003;376(3): 615-23.

49. Wang J, Wang CE, Orr A, Tydlacka S, Li SH, Li XJ. Impaired ubiquitinproteasome system activity in the synapses of Huntington's disease mice. J Cell Biol. 2008;180(6):1177-89.

50. Plafker SM. Oxidative stress and the ubiquitin proteolytic system in age-related macular degeneration. Adv Exp Med Biol. 2010;664:447.

51. Shin SW: (the) inducible immune genes against invading microorganisms in the fall webworm, Hyphantria cunea. 2000.
52. Asea A, Rehli M, Kabingu E, Boch JA, Baré O, Auron PE, Stevenson MA Calderwood SK. Novel signal transduction pathway utilized by extracellula HSP70 ROLE OF toll-LIKE RECEPTOR (TLR) 2 AND TLR4. J Biol Chem. 2002; 277(17):15028.

53. Vabulas RM, Ahmadnejad P, Da CC, Miethke T, Kirschning CJ, Häcker H, Wagner $\mathrm{H}$. Endocytosed HSP60s use toll-like receptor 2 (TLR2) and TLR4 to activate the toll/interleukin-1 receptor signaling pathway in innate immune cells. J Biol Chem. 2001;276(33):31332-9.

54. Roelofs M, Boelens W, Joosten L, Berg WD, Radstake T: Identification of small heat-shock protein B8 (HSP22) as a novel toll-like receptor 4 ligand and potential involvement in the pathogenesis of rheumatoid arthritis. 2006.

55. Shukla AK, Pragya P, Chaouhan HS, Tiwari AK, Patel DK, Abdin MZ, Chowdhuri DK. Heat shock Protein-70 (Hsp-70) suppresses Paraquatinduced Neurodegeneration by inhibiting JNK and Caspase-3 activation in drosophila model of Parkinson's disease. PLoS One. 2014:9(6):e98886.

56. Gong M, Li YJ, Dai X, Tian M, Li ZG. Involvement of calcium and calmodulin in the acquisition of heat-shock induced thermotolerance in maize seedlings. J Plant Physiol. 1997;150(5):615-21.

57. Jiang N, Tan X, Zhang L. Gene analysis of Phosphatidylinositol signaling system in fatty Acide developing of Camellia Oleifera seeds based on RNA-Seq. J Food Sci Biotechnol. 2014;33(6):583-9.

58. Borggrefe T, Oswald F. The notch signaling pathway: transcriptional regulation at notch target genes. Cell Mol Life Sci. 2009;66(10):1631-46.

59. Wang H, Kiuchi T, Katsuma S, Shimada T. A novel sucrose hydrolase from the bombycoid silkworms Bombyx Mori, Trilocha varians, and Samia cynthia ricini with a substrate specificity for sucrose. Insect Biochem Mol Biol. 2015: 61:46-52.

60. Xiao R, Wang L, Cao Y, Zhang G. Transcriptome response to temperature stress in the wolf spider Pardosa Pseudoannulata (Araneae: Lycosidae). ECOL EVOL. 2016;6(11):3540-54.

61. Moallem Z, Karimimalati A, Sahragard A, Zibaee A. Modeling temperaturedependent development of Glyphodes pyloalis (Lepidoptera: Pyralidae) J Insect Sci. 2017:17(1):1-8.

62. Yang B, Huang W, Zhang J, Xu Q, Zhu S, Zhang Q, Beerntsen BT, Song H, Ling $\mathrm{E}$. Analysis of gene expression in the midgut of Bombyx Mori during the larval molting stage. BMC Genomics. 2016;17(1):866.

63. Pertea G, Huang X, Liang F, Antonescu V, Sultana R, Karamycheva S, Lee $Y$, White J, Cheung F, Parvizi B. TIGR gene indices clustering tools (TGICL): a software system for fast clustering of large EST datasets. Bioinformatics. 2003;19(5):651-2

64. Li X, Shi L, Zhou Y, Xie H, Dai X, Li R, Chen Y, Wang H. Molecular evolutionary mechanisms driving functional diversification of a-glucosidase in Lepidoptera. SCI REP-UK. 2017;7:45787.

65. Dunning LT, Dennis AB, Park D, Sinclair BJ, Newcomb RD, Buckley TR. Identification of cold-responsive genes in a New Zealand alpine stick insect using RNA-Seq. Comp Biochem Physiol Part D Genomics Proteomics. 2013;8(1):24-31.

66. Teets NM, Denlinger DL. Gene expression changes governing extreme dehydration tolerance in an Antarctic insect. P NATL ACAD SCI USA. 2012 109(50):20744.

67. Livak KJ, Schmittgen TD. Analysis of relative gene expression data using real-time quantitative PCR and the 2(-Delta Delta $C(T))$ method. Methods. 2001;25(4):402-8.

\section{Submit your next manuscript to BioMed Central and we will help you at every step:}

- We accept pre-submission inquiries

- Our selector tool helps you to find the most relevant journal

- We provide round the clock customer support

- Convenient online submission

- Thorough peer review

- Inclusion in PubMed and all major indexing services

- Maximum visibility for your research

Submit your manuscript at www.biomedcentral.com/submit 